\title{
Attogram dust grains in the solar system
}

\section{Forming mechanism, properties and dynamics}

\author{
V. P. Vasylyev ${ }^{1}$, A. I. Kalinichenko ${ }^{2}$, and S. V. Vasylyev ${ }^{3}$ \\ 1 SOLERC, PO Box 30, Kharkov, 61052, Ukraine \\ e-mail: vicv@vil.com.ua \\ 2 Kharkov National University, PO Box 60, Kharkov, 61052, Ukraine \\ e-mail: Alex.I.Kalinichenko@univer . kharkov .ua \\ 3 SVV Technology Innovations, Inc., PO Box 375, West Sacramento, CA 95691, USA
}

Received 27 March 2001 / Accepted 23 December 2003

\begin{abstract}
In this paper we conceptually develop the theory of attogram dust grain formation by the cosmic-ray ion-enhanced brittle microerosion of atmosphereless solar system bodies. According to our theory, the multicharged cosmic ray ions penetrating into the solid material of planetary satellites, asteroids, comets and smaller space bodies give rise to microscopic cylindrical overheated zones and generate mechanical impulses and thermoelastic stresses exceeding the material tensile strength. The impulses may cause micro-scale shattering of the surfaces and the ejection of ultrafine (attogram) dust particles off the parent body. The attogram grains formed by this mechanism (the so-called track-breaking mechanism) have an average mass of $10^{-19}$ $10^{-17} \mathrm{~g}$, a very flaky shape and ejection velocities of from tens to hundreds $\mathrm{m} \mathrm{s}^{-1}$. Depending on the ejection velocity and a large array of non-gravitational forces, such grains can deposit and accumulate on the parent body surface, orbit around the body thus forming a bound dust cocoon, or finally, escape the body as ultrafine-dust wind. Quantitative data on dust contributions to these varieties of attogram population depending on the sizes and parameters of parent body material are obtained and discussed. The volumetric track-breaking resulting in attogram grain deposition in the ice-rock mixture of cometary nuclei during the whole period of their formation is analysed. Taking the example of Halley's comet we demonstrate that the mechanism under study makes it possible to quantitatively explain the in situ measured considerable excess of attogram grains within the cometary environment in its circum-solar path sections. The problem of track-breaking disintegration of small-sized bodies in the Kuiper Belt, which are permanently subjected to a cosmic-ray ion-enhanced brittle microerosion heavier than in the Main Belt, is investigated. The track-breaking survival timescale for submicron and over hundred micron-sized grains is found to be considerably smaller than the escape timescale of the Poynting-Robertson and plasma drags, as well as the collisional mechanism. Track-breaking can also be competitive to the collisional escape in the range of grain sizes of 1 to 100 microns. A quantitative analysis of the calculations shows that the present theory may adequately explain both the observable micron/submicron dust grain depletion of the Kuiper Belt and the loss of its bulk mass during evolution. Some possible observational tests for the future revealing the predicted populations of attogram dust in the solar system are also discussed.
\end{abstract}

Key words. comets: general - comets: individual: Halley - solar system: general - interplanetary medium

\section{Introduction}

In situ detection of attogram dust grains (ADGs) in the proximity of Halley's comet (Sagdeev et al. 1989; Utterback \& Kissel 1990; van Nes 1986) proves the existence in the solar system of a formerly unknown dust population which is halfway between the micron/submicron-sized grains and the molecular component. The presence of this population was also indicated (see, e.g., Krasnopolsky 1997) by the observational data on the X-ray emission of the Hale-Bopp (Lisse et al. 1997) and Hyakutake (Lisse et al. 1996) comets and, probably, by

Send offprint requests to: $\mathrm{S}$. V. Vasylyev,

e-mail: vasilyev@svvti.com the blue excess in (2060) Chiron's dust coma (West 1991). The data can be interpreted if the existence of a representative attogram fraction in the size distribution spectrum of dust grains is assumed. The experimentally studied lunar-rock impact microcraters also indicate the presence in the solar system of dust grains with masses of tens of attograms and less (Hutcheon 1975).

According to laboratory-scale experiments (see, e.g., Eichhorn 1975), relatively larger grains may be of impact origin. This makes it possible to explain the existence of microcraters with diameters of about 0.1 microns and larger as due to the secondary impact of minute debris produced by the primary micrometeoroid impacts. However, the smallest impact 
nanocraters with diameters about one hundredth of a micron and smaller can be formed purely by the attogram dust grains with masses circa $10^{-18} \mathrm{~g}$ and less, i.e. similar to those detected near Comet Halley. There are no proofs for the existence of such ultrasmall debris in the products of micrometeoroidal impacts, which points to low probability of their impact origin.

On the other hand, the origin and properties of the nanoparticle component of interstellar dust (PAHs as well as silicon and carbonaceous grains) are being intensively studied (see, e.g., $\mathrm{Li}$ $\&$ Draine 2001, and references therein). The existing models and mechanisms for the formation and evolution of interstellar dust grains, which consider their formation from submolecular sizes as well as their shattering, particularly, by grain-grain collisions and interstellar shocks, implicate the presence in the interstellar medium of a representative population of ADG with grain sizes down to $10^{-7} \mathrm{~cm}$ (see, e.g., Tielens 1999). The sizes typical for the observed interstellar dust grains are considerably smaller than for the interplanetary ones. This agrees with the conjecture of an interstellar nature of cometary ADG (see, e.g., Sagdeev et al. 1989). However, the conditions enabling the intense formation of ADGs actually exist in the solar system too, as well as in other planetary/protoplanetary systems. Earlier (Kalinichenko et al. 1996) we showed that the so-called track-breaking can be such a formation mechanism.

Qualitatively, the mechanism consists of the following. High-energy multicharged ions of galactic and solar cosmic rays interact with the surfaces of atmosphereless solar system bodies (ASSB) causing microerosion on the latter by shattering the ultra-fine solid fragments and ejecting them into the interplanetary medium. As the ionization energy loss density near the heavy multicharged ion path in a substance can reach several electron-volts per atom, equivalent to tens of thousands $\mathrm{K}$ overheating, the corresponding thermoelastic stresses in the track $(\sim 10 \mathrm{GPa})$ considerably exceed the dynamic tensile strength of typical ASSB materials (0.01-1 GPa). Rapidly originating thermoelastic stresses in an ion track are taken by the ASSB material as sui generis thermal impact, showing up in the formation of a divergent cylindrical acoustic stress wave. The interaction of such a wave with the ASSB surface adjoining space or a material with smaller acoustic rigidity may result in microscale shattering of the surface and solid fragments being chipped off. The smallest size of the shattered fragments is determined by the effective radius of the overheated region (energetic track), which varies for different ASSB materials from 10 to 100 angströms. The largest fragment size is determined by the length of ion track projection on the surface plane from the entry point to the point where the amplitude of an acoustic wave reaching the surface corresponds to the material ultimate strength. A typical fragment has the shape of a flake or a scale, whose different points acquire different velocities in shattering. This may result in its further disintegration into attogram masses, viz. ADG formation.

We present further development and a theoretical analysis of track-breaking mechanism and its effects to allow qualitative and quantitative assessments of the present knowledge about ADGs. It also aims at paving the way for the search for new observational evidence of this type of dust population in the solar system.

\section{Surface track-broken attogram dust grains}

For a more in-depth study of the surface track-breaking mechanism let us define the geometrical and kinematic adjectives of an ASSB fragment shattered in the interaction of a cosmic ray ion with the surface material, employing the model from Kalinichenko et al. (1996). Consider the interaction of an axial-symmetrical elastic wave excited in the ion track with an ASSB-space plane boundary. The amplitude-time characteristics of this wave are determined by the radial distribution of the ion energy losses and by the rate of their relaxation into the temperature of the ASSB material. In turn, the duration of an ionization energy relaxation into this temperature depends on the electron properties of the material. Considering purely dielectric materials, assume that the temperature transient period being determined by the ion-ion relaxation time $t_{\mathrm{ii}} \approx 10^{-14} \mathrm{~s}$. As the value of $t_{\mathrm{ii}}$ is relatively small compared to the time of acoustic relaxation of an energy ion track, the power function of heat sources $Q(r, t)$ in this track can be represented in the description of thermoacoustic oscillation excitation as

$Q(r, t)=\tau_{\mathrm{ii}}^{-1} \exp \left(-\frac{t}{\tau_{\mathrm{ii}}}\right) \varepsilon(r) \chi(t)$,

where $\varepsilon(r)$ is the ionization energy density in a multicharged ion track, $\chi(t)$ is the Heaviside unit step function. Eventually, the axisymmetric thermal field (1) that comes into being almost immediately results in thermoelastic stresses, which show up as the impulse load distributed over a length of the ion track and generating a divergent elastic cylindrical wave in the material.

The comparison of the calculated amplitude of the thermoelastic stresses generated by the thermal field (1) with the amplitude of stresses generated by the cylindrical thermal field with a radius of $R_{\mathrm{c}} \approx 10^{-9} Z^{2} \rho^{-1}$ and including the $k=0.6$ quantity of the primary-particle energy losses ( $Z$ and $\rho$ are the effective average charge and the ASSB material density, respectively) shows the that they are very similar, as are the positive halfwave periods. The positive half-wave of a thermoelastic impulse contributes most to the ASSB surface ion-enhanced erosion mechanism. Therefore, as in Kalinichenko et al. (1996), from now on we will use the characteristics of the model-track generated impulse. The model track, or the so-called energetic track is a uniformly heated cylindrical region with radius $R_{\mathrm{c}}$ and containing the bulk of the energy losses of a multicharged ion penetrating under the surface.

The estimated magnitudes of $R_{\mathrm{c}}$, overheating temperatures $T_{\mathrm{c}}$ and thermoelastic stresses $\sigma_{\mathrm{c}}$ originating in the energetic track are given in our earlier work Kalinichenko et al. (1996).

As is also shown there, the ASSBs of different sizes can be subdivided into three classes depending on the different behavior and contribution to the attogram dust population of their ADGs. Let us analyze this contribution quantitatively for each of the ASSB classes. For this purpose, the concept of an erosion zone (EZ) formed on the ASSB plane surface will be employed, see Fig. 1 in Kalinichenko et al. (1996).

With an average EZ volume of about $10^{-15} \mathrm{~cm}^{3}$ the EZ destruction due to the surface shattering of the parent body generates ADGs with characteristic masses of about $10^{-19}-10^{-17} \mathrm{~g}$. 


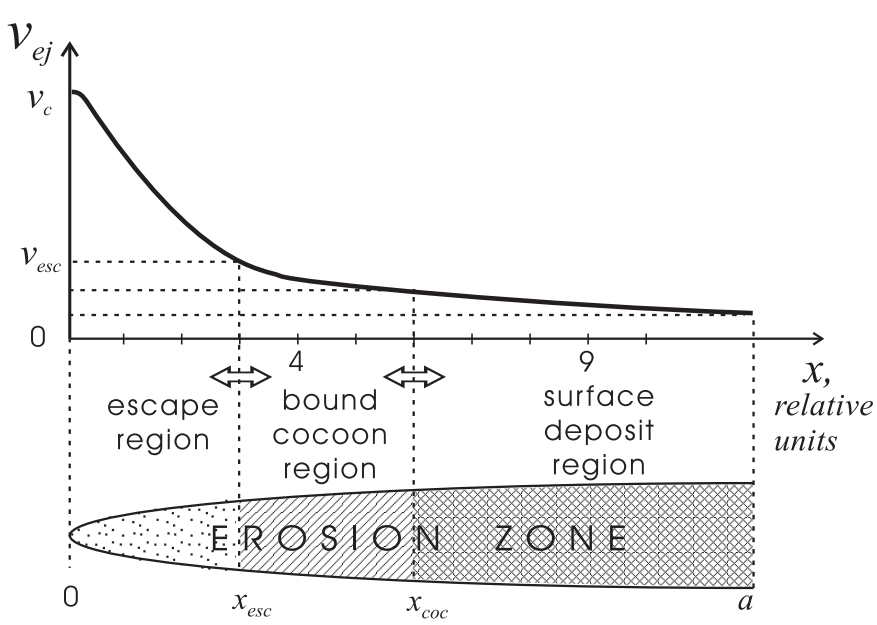

Fig. 1. The principle of EZ division by subzones which, depending on the proportion between the parent body size and the attogram fragment ejection velocity $v_{\mathrm{ej}}$, yield different components of the ADG population (arbitrary scale). The top of the figure shows the qualitative curve corresponding to the $v_{\mathrm{ej}}$ dependence of the EZ fragment on the distance to the point, where a multicharged ion enters the ASSB material.

Seeing that the high-energy multicharged ions of primary cosmic rays fill the near-ASSB space isotropically, this process is going on continuously practically everywhere in the solar system. In other words, almost all ASSBs are parent bodies for the continuously replenished ADG population. The ASSB division in three classes results from the fact that ADGs, being EZ fragments, in track-breaking acquire different velocities $v_{\mathrm{ej}}$ (see the qualitative curve at the top of Fig. 1).

Now let us analyze how these velocities correlate with the location of a fragment in EZ, as this relationship will be decisive in the search for observational evidence in favor of the discussed theory.

Formally, the proportion between $v_{\text {ej }}$ and $v_{\text {esc }}$ is set by the space boundaries of different EZ subzones which supply the ultra-fine dust to free space, to the cocoon, and to the ASSB surface deposited layer, respectively. Depending on the average radius $R_{\mathrm{ASSB}}$ and on the ASSB average density $\bar{\rho}$, the boundaries with coordinates $x_{\text {esc }}$ and $x_{\text {coc }}$ (see Fig. 1), which break up the EZ into subzones, may take various loci. On the other hand, due to the dependence of escape velocity $v_{\mathrm{esc}}$ on $R_{\mathrm{ASSB}}$, the discussed model suggests that in terms of ADG dynamics there exist two critical values of the parent-body average radius: $R_{\mathrm{ASSB}}^{\min }$ and $R_{\mathrm{ASSB}}^{\max }-$ see Fig. 2 . This figure shows the quantitative calculation of the attogram dust population contribution to the described varieties versus $R_{\mathrm{ASSB}}$, assuming a possible transversal effect of various forces after the grains are shattered from the parent body. The dynamic peculiarities of ADGs for different ASSB classes are shown schematically in Fig. 3.

The evaluations made for the model where only the light pressure is considered show that the ADGs escaping the surface of a Chiron-like asteroid in the direction normal to its unshaded-area surface have a very high chance to be captured into a bound orbit. To enable such behavior of ADGs, their initial velocities should be less than $v_{\text {esc }}$ by about a factor 100 . With this in mind the Fig. 2 data on the relative proportion of

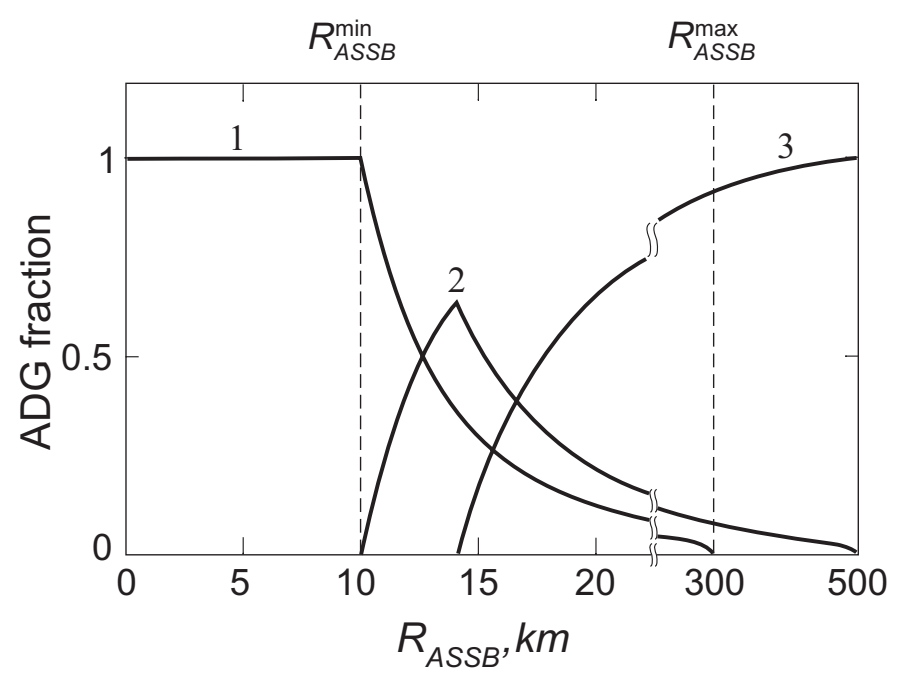

Fig. 2. The calculated dependence of ASSB size on the contribution of the ADGs, yielded by ASSB surface ion-enhanced microerosion into different types of the attogram dust population: dust wind (curve 1), bound cocoon (curve 2) and deposited/accumulated layer (curve 3). The calculation was made for the ASSBs whose material parameters approximately correspond to the known characteristics of asteroids, for $\rho=\bar{\rho}$, and without taking non-gravitational forces into account.

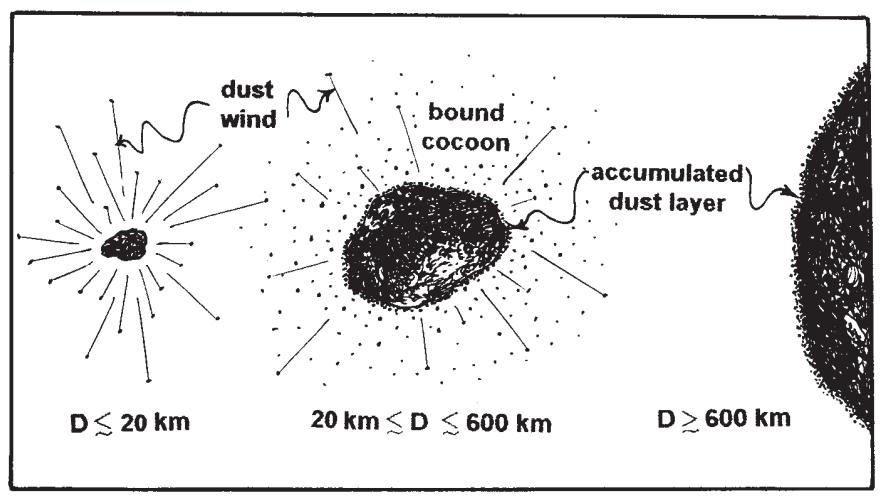

Fig. 3. Three types of parent ASSBs capable of producing the ADGs revealing different types of dynamic response shown in correspondence with the calculations from the previous figure.

the ADGs hitting the bound cocoon can be considered an upper estimate.

The estimates and Fig. 2 show that the range of sizes of asteroid-like ASSBs capable of forming a dust cocoon is rather broad, varying from 20 to $600 \mathrm{~km}$. Within this range, the ASSBs of about $30 \mathrm{~km}$ in size are most likely to develop dust cocoons; for the moment we will disregard their rotation. However, with low-density material (e.g., fluffy ice-dust-rock mixture) this size can increase by a large factor. Specifically, the HST observations give density parameters for the Centaur 2060-Chiron of about $0.4 \mathrm{~g} \mathrm{~cm}^{-3}$ (Meech 1994) corresponding to just such a case. This makes it possible to assume that trackbreaking makes an essential contribution to the formation of a cocoon-like dust population such as may be observable around some ASSBs. We can also assume that we may already be observing this population for 2060 Chiron, though it is often referred to as a coma by analogy to comets even though it is 
questionable that evaporation/sublimation of volatile matter is the case here.

The size increase of the ASSBs which most probably have such a cocoon can be explained by their rotation. However, this will have no impact on the total ADG production. The increase is the result of the redistribution of the number of ADGs yielded by different regions of an EZ subzone (see Fig. 1), which, in turn, results from the increase of the effective value of $v_{\mathrm{ej}}$. The estimates show, for example, that with an asteroid rotation period of several hours, such rotation should already be taken into account in defining the final value of $R_{\mathrm{ASSB}}^{\min }$ for the equatorial zones of an asteroid. All this means that the "low density + rotation" combination shifts the size range of ASSBs, whose ADG-comprising bound cocoon may most probably be observed, to hundreds of kilometers. There is exactly one known object in this range, viz. 2060 Chiron. Therefore, when searching for ADG cocoons, ASSBs in the range of thirty (high average density and slow rotation) to several hundred (low average density and faster rotation) kilometers give the best prospects. The ADGs may either be free-flying in the cocoon or form clusters, thus producing larger light-scattering grains.

In fact, the ADGs shattered from the parent body are exposed to a variety of nongravitational forces. A quantitative study of the further dynamic behavior of these particles and the properties of the bound cocoon consisting of track-broken ADGs is an extremely complicated problem where many factors should be considered. Among these mostly unknown factors, are the ADG initial velocity value and direction, the ADG shape and size, its dielectric and magnetic properties, its charge, the size, density and rotation parameters of the parent body, the ADG path shadowing, etc. Therefore, in this paper, in order to give a firm base for the possible existence of such ADG accumulation mechanism in a cocoon, we will consider the two-body problem, taking into account the solar radiation pressure only.

In defining the ADG repopulation rate in the solar system, the specific mass of the EZ fragments never returning to the parent ASSB should be estimated. Such an escape mass for non-icy ASSBs is

$m_{\mathrm{esc}} \approx \frac{4 \sqrt{2}}{3} \rho R_{\mathrm{c}}^{3}\left(\frac{R_{\mathrm{ASSB}}^{\max }}{R_{\mathrm{ASSB}}}\right)^{3} \ln \frac{l}{r_{\max }}$,

where, according to Kalinichenko et al. (1996), the values $l$ and $r_{\max }$ are functions of the parameters of the multicharged cosmic ray ions and of ASSB material. Figure 3 shows that the EZ mass, which supplies the ADGs in a cocoon, has its maximum value with $R_{\mathrm{ASSB}} \sim 14 \mathrm{~km}$ for non-icy ASSBs and disregarding their rotation. This value can be found using the formula

$m_{\mathrm{coc}}^{\max } \approx \frac{1}{12}\left(2^{3 / 2}-1\right) \rho R_{\mathrm{c}}^{3}\left(\frac{\sigma_{\mathrm{c}}}{\sigma_{\mathrm{d}}}\right)^{3} \ln \frac{l}{r_{\max }}$.

By inserting the following estimates: $r=3 \mathrm{~g} \mathrm{~cm}^{-3} ; R_{\mathrm{c}}=$ $1.2 \times 10^{-7} \mathrm{~cm} ; \sigma_{\mathrm{c}}=8.26 \mathrm{GPa} ; \sigma_{\mathrm{d}}=0.1 \mathrm{GPa} ; \ln l / r_{\max }=8$ we obtain for the maximum EZ mass contributing to a cocoon in each instance of track-breaking $m_{\text {coc }}^{\max } \sim 3.5 \times 10^{-15} \mathrm{~g}$. For $m_{\text {esc }}$ with the same parameters we obtain a value of the order of $1.9 \times 10^{-15} \mathrm{~g}$. Thus, in typical conditions a significant amount of the EZ $\left(\sim 5 \times 10^{-15} \mathrm{~g}\right)$ repopulates the ASGs in each solitary instance of track-breaking of asteroidal-size ASSB surfaces subject to ion-enhanced erosion. With these values of the specific mass and flux of multicharged ion cosmic rays, the model used makes it possible to evaluate the absolute rate of ADG repopulation for a given ASSB class. However, the problem of the contribution to this population by the different classes of ASSBs versus their position in the solar system is rather complicated and requires a special analysis for each specific body. This work is limited to a more detailed consideration of the contribution to ADG production of comets approaching the Sun for which a quantitative comparison can be made with the observed data obtained in the proximity of Halley's comet. We consider also in detail the small-size bodies in the Kuiper Belt where, as far as we know, such bodies contain the bulk of the mass.

\section{Comet-produced ADGs}

The most conclusive evidence in favour of the existence of ADGs in the solar system is currently provided by "Vega" (Sagdeev et al. 1989) and "Giotto" (Utterback \& Kissel 1990) space-borne direct measurements made in the proximity of Halley's comet, the X-ray observation data for the Hyakutake and Hale-Bopp comets (Lisse et al. 1996, 1997), and the presence of ultra-fine impact craters in the lunar rocks (Hutcheon 1975). Quantitative analysis of the measurements made at distances of about 1 million $\mathrm{km}$ from the nucleus of Halley's comet shows that the actual concentration of ADGs corresponds to $6 \%$ of the total mass ejected from the nucleus. This exceeds by several orders the value obtained in Lamy et al. (1987) by the extrapolation of an experimental value of the mass distribution of dust particles for masses from $10^{-15} \mathrm{~g}$ and more. This is a non-trivial result that has not yet been adequately interpreted, but it can be explained by a modified mechanism of track-breaking of the ice-rock/dust mixture that forms the cometary nuclei.

The basic assumptions for this modified model, i.e. for the volumetric track-breaking, are as follows. Firstly, the model must account for the natural fact that bombardment by cosmic ray multicharged ions, and hence, track-breaking excavation of cometary nuclei, occur during the whole evolution of the nuclei - from the origin of cometesimals to the present sizes and orbits of the nuclei. Secondly, the bicomponent material of a cometary nucleus consisting of rocky fragments with a size distribution $f(R)=B \cdot R^{-4}$ and existing in an icy matrix, which fills all the space between the fragments (see Fig. 4), is assumed to be continuously subjected to track-breaking. We assume that we can approximate the rocky fragments by spherical solids with a minimum radius of 0.1 micron. Then, with allowance for a weak (logarithmic) dependence on the maximum size of rock fragments, and with equal volume fractions of rock and ice, the $B$ constant takes on a value of about 0.03 . In such a model, the surface layer in a cometary nucleus is not only modified due to evaporation (Luu 1994), but also, near perihelium, converted from an ice-rock mixture into a dust-rubble mantle, owing to ion-enhanced erosion of the icy component and to the saturation of the ASSB material with ADGs. Figure 5 schematically 


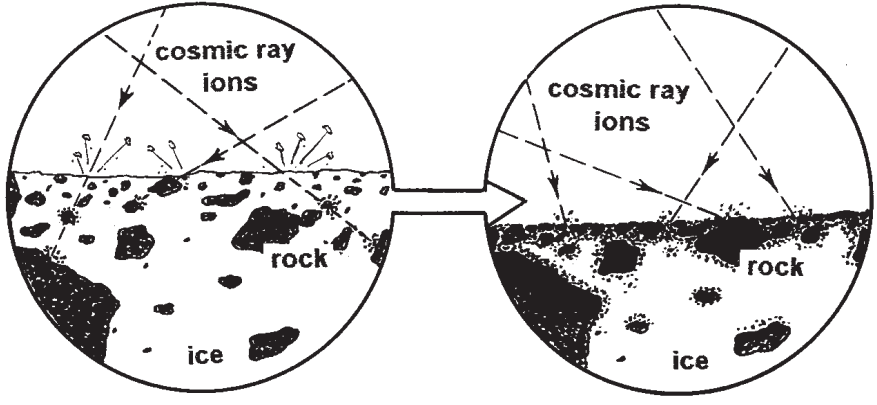

Fig. 4. A schematic diagram showing the formation of a dust-rubble mantle in the surface layer of a cometary nucleus. Owing to a much more intensive ion-enhanced microerosion of the icy component in the ice-rock mixture, the nucleus surface layer converts into an ADGsaturated rubble mantle. This process, in contrast to rubble mantle formation due to evaporation and sublimation of volatiles (Luu 1994), becomes more efficient at remote distances from the Sun.

shows this process, whose intensity increases with the distance from the Sun. Through the entire evolution of the nucleus the ADGs are mainly formed not on the "rock-space" interface but on the inner "rock-ice" interface. This is due to the fact that the heterogeneous medium of a cometary nucleus consisting of components with quite different acoustic stiffness, such as rock and ice, offers more favorable conditions for track-breaking when an elastic wave generated by an ion energetic track propagates through it.

For quantitative estimations in the described model we employ the concept of a destruction zone (DZ) introduced and analysed in Vasylyev et al. (1998). Firstly, determine the volume of the DZ bounded in Fig. 5 by a dashed line. Let $l$ be a coordinate along the axis of the ion energetic track counted from the ion entry point, and let $r_{\mathrm{d}}(l)$ be the DZ radius as a function of $l$. The DZ volume is given by the expression

$V_{\mathrm{d}}=\int_{0}^{L_{\mathrm{d}}} \pi\left[r_{\mathrm{d}}(l)\right]^{2} \mathrm{~d} l$,

here $L_{\mathrm{d}}$ is the DZ effective length that practically coincides with the energetic track length in the material of a cometary nucleus. For an estimate, we may take the value of $r_{\mathrm{d}}(l)$ corresponding to the initial part of the energetic track far from the breaking point. For an ion with an energy of $0.2 \mathrm{GeV} /$ nucleon, a charge of $z=25$ and with $\rho=2 \mathrm{~g} \mathrm{~cm}^{-3}$ for the ice-rock mixture material, the estimate will give $r_{\mathrm{d}} \sim 10^{-5} \mathrm{~cm}, L_{\mathrm{d}} \approx 85 \mathrm{r}^{-1} A^{-1} \approx 0.85 \mathrm{~cm}$. In this approximation we obtain $V_{\mathrm{d}} \approx 2.7 \times 10^{-10} \mathrm{~cm}^{3}$. For the volume fraction $\Delta v_{\mathrm{ADG}}$ of DZ-yielded attogram grains with a size distribution $f(R)=0.03 \cdot R^{-4}$ the following estimation is valid:

$\Delta v_{\mathrm{ADG}}=\int_{R_{\min }}^{R_{\max }} f(R) 4 \pi R^{2} c \mathrm{~d} R \approx 3.6 \times 10^{-3}$

for $R_{\min }=0.1 \mu \mathrm{m}$. Accordingly, for the total volume of the ADGs yielded in the DZ of a single multicharged ion we obtain $\Delta V_{\mathrm{ADG}}=\Delta v_{\mathrm{ADG}} V_{\mathrm{d}} \sim 10^{-12} \mathrm{~cm}^{3}$. Comparison of the obtained $\Delta V_{\mathrm{ADG}}$ value with the $\mathrm{EZ}$ volume shows that the mechanism of volumetric track-breaking of rock in the ice-rock

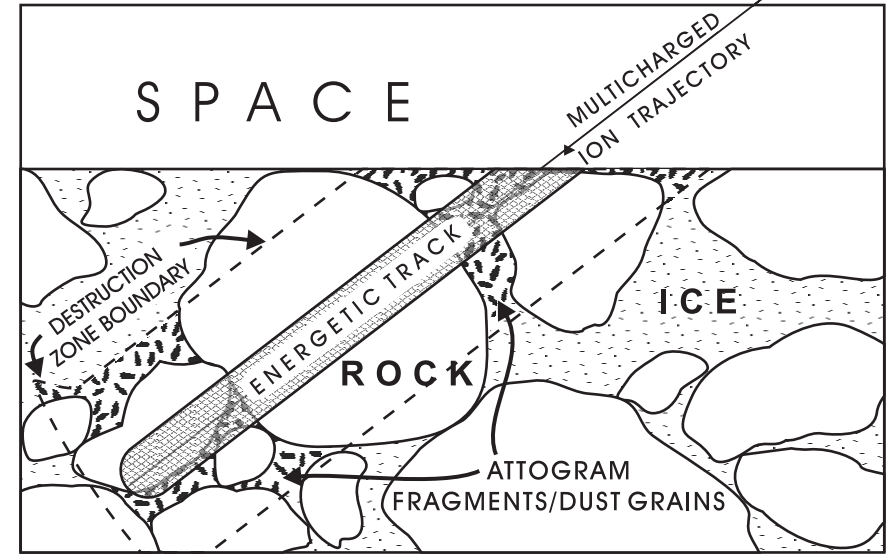

Fig. 5. The volumetric modification of the track-breaking model in which the ice-rock/dust mixture of which the cometary nuclei consist are bombarded by multicharged cosmic-ray ions. The ADGs, remaining embedded in an icy matrix till they are ejected during ice evaporation on the circumsolar path of a comet trajectory, have a high formation rate in the destruction zone (bounded by the dashed line) of the subsurface layer in the nucleus formed of cometesimals.

mixture is more effective by 2 to 3 orders of magnitude than the near-surface ion-enhanced erosion of "monolithic" rock-type materials.

Therefore, when the whole volume of a cometary nucleus is excavated in track-breaking resulting in ADG accumulation through the whole formation period of the nucleus, these ADGs can be ejected with the volatile matter during the perihelion passage of the comet. Assuming this period to be equal to $10^{8}$ years, for a nucleus radius to reach $10 \mathrm{~km}$ will require a rate of growth of about $10^{-4} \mathrm{~m}_{\text {year }}{ }^{-1}$. Then the proportion of the attogram dust fraction in the material of a comet nucleus $v_{\mathrm{ADG}}$ is determined by the total ADG production due to volumetric track-breaking for the whole period of nucleus formation:

$\nu_{\mathrm{ADG}} \approx 2 \pi J \frac{10^{8} \text { years }}{10 \mathrm{~km}} \Delta V_{\mathrm{ADG}}$.

Provided a value of $J \approx 0.03 \mathrm{~cm}^{-2} \mathrm{~s}^{-1} \mathrm{sr}^{-1}$ is taken for the flux of the superheavy component of cosmic ray multicharged ions beyond the solar system, we obtain $v_{\mathrm{ADG}} \approx 7 \times 10^{-2}$, assuming Eq. (5) is valid and taking the parameters of the nucleus given above. This estimate is very close to the relative mass of ADGs measured near Halley's comet. Thus, for about a 3-month period of comet motion around perihelium, when the comet loses about $10^{9}$ tons of its mass (corresponding to the loss of a layer from the nucleus of circa $0.2 \mathrm{~m}$ ), about $7 \times 10^{7}$ tons of the nucleus material can be ejected as ADGs.

An interesting feature of the ADG flux measured in the proximity of Halley's comet is the ADG mass distribution. According to Utterback \& Kissel (1990) the exponent in the function of integral ADG-distribution is somewhere between unity, which is characteristic of dust of impact nature, and two. This knowledge can be employed to verify the suggested formation mechanism of ADGs as well. Let us utilize the two features which describe the track-breaking process. The first is the constancy in thickness $\left(c \sim R_{\mathrm{c}}\right)$ of the flaky ADGs formed in track-breaking. The second is the probable correspondence 
of the effective cross section of the ADG to the distribution of EZ surface irregularity sizes. These irregularities are, in turn, the effect of the excavation of the ASSB surface by impacts. In this case $f(a) \sim$ const $\cdot a^{-4}$, where $a$ is the effective cross-section of the ejected EZ fragment. Then the differential ADG mass distribution $\mathrm{d} N / \mathrm{d} m \sim$ const $\cdot m^{-5 / 2}$ will differ from that for the particles that are due to impacts. Eventually, we obtain for the amount of ADGs with mass exceeding $m$ : $N_{\mathrm{ADG}}(>m) \sim$ const $\cdot m^{-3 / 2}$.

The agreement between the quantitative estimations of the mass distribution and the experimental evidence indicates that the model we have considered is valid. It also supports our interpretation of the measurements made in the proximity of Halley's comet and estimates of the total ADG amount produced by all cometary nuclei in large. Note that the considered mechanism agrees well with the new analysis of the formation of cometary nuclei (Greenberg 1998), where a substantial amount of ADG is embedded in the icy fraction of the nucleus.

As a comparison shows, none of the previously known mechanisms (endogenous or exogenous, including the surface type of track-breaking) is able to ensure this production and accumulation of attogram dust grains in the material of the cometary nucleus. In this regard, no difference can be observed between long-period and short-period comets in the partial density of attogram particles in their dust environments.

\section{ASSB survival and yielded ADGs in the Kuiper Belt}

The Kuiper Belt (KB) lies at a distance of from $30 \mathrm{AU}$ to $50 \mathrm{AU}$ from the Sun and contains at least $10^{5}$ objects with diameters above $100 \mathrm{~km}$ (Jewitt 1999). From the size distribution function $\sim R^{-4}$ it follows that the $\mathrm{KB}$ contains about $10^{11}$ objects with sizes $>1 \mathrm{~km}$, as well as a large amount of small dust. The current estimate of the total mass of micron-sized particles only in the $\mathrm{KB}$ gives about $8 \times 10^{16} \mathrm{~kg}$ (Curnett et al. 1997). Note that the last estimation, based on Voyager 1 data measured in situ, falls short of the value one expects using the size distribution function $\sim R^{-4}$. This may indicate that the KB dust has a limited survival time. The discussed properties of the KB, as well as the fact that the KB is quite far away from the sun and thus is subjected to a more effective ion-enhanced erosion due to a weaker contribution of the cosmic ray energy spectrum truncation mechanism, make it possible to take the $\mathrm{KB}$ as the major ADG-contributor to the solar system.

Consider now the problem of the intensity of formation of ADG in the KB by analyzing the problem of the survival of the smallest ASSBs, which possess the largest overall surface area, and are bombarded by multicharged ions. The analysis shows that the rate of the ion-enhanced brittle surface erosion for these bodies, and thus, their survival time $t_{\mathrm{s}}$ and the specific intensity of the ADG-generated dust wind essentially depend on the $R_{\mathrm{ASSB}}$ to DZ-size ratio. Based on the analogy of the previous section, the evaluated DZ length $L_{\mathrm{d}}$ and DZ radius $r_{\mathrm{d}}$ give values for the rock material of circa $0.6 \mathrm{~cm}$ and $10^{-5}$ $10^{-4} \mathrm{~cm}$, respectively. Another characteristic size, defining the $t_{\mathrm{S}}$ of the smallest particles - from the micron-sized grains to the ADGs, is the minimal effective radius $R_{\text {min }}$, which as estimated in Kalinichenko et al. (1996) is about $2 \times 10^{-7} \mathrm{~cm}$. Grains of this size and smaller may completely evaporate even in a single interaction with a multi-charged cosmic ray ion. On the other hand, their cross section is extremely small, giving an very low frequency of interaction. Therefore the value of $t_{\mathrm{s}}$ for dust particles with the effective radius less than $R_{\min }$ is inversely proportional to the square of the radius of these ultrafine grains: $t_{\mathrm{S}} \approx 1 / 4 \pi^{-2} J^{-1} R_{\mathrm{ASSB}}^{-2}$. We should emphasize that for $R_{\mathrm{ASSB}}>R_{\min } t_{\mathrm{s}}$ is the time for which all ASSB will be trackbroken down to the ADG sizes. For the smallest dust grains $\left(<R_{\min }\right) t_{\mathrm{s}}$ is the time until vaporisation in a collision with a multicharged ion.

In the range $R_{\min }<R_{\mathrm{ASSB}}<r_{\mathrm{d}}$, for any collision with a multicharged ion, the mechanical stresses at any point of the grain surface exceed the dynamic tensile strength of the grain material. In other words, the EZ area coincides with the overall surface area of such ASSBs, thus their disintegration is extremely rapid. In this case, the rate of ion-enhanced disintegration versus grain radius, assuming that the ASSB approximate a spherical shape, can be found to be

$-\frac{\mathrm{d} M\left(R_{\mathrm{ASSB}}\right)}{\mathrm{d} t} \approx 16 \pi^{3} R_{\mathrm{ASSB}}^{4} \cdot J \cdot c \cdot \rho$.

After integration we obtain the survival time of the micronsized and smaller particles

$t_{\mathrm{S}}\left(R_{\mathrm{ASSB}}\right) \approx \frac{1}{4 \pi^{2} J \cdot c}\left(R_{\mathrm{min}}^{-1}-R_{\mathrm{ASSB}}^{-1}\right)$.

From formula (8) it follows that, in this size range, the $t_{\mathrm{S}}$ depends only weakly on the particle radius, and for $R_{\mathrm{ASSB}}>$ $2 \times 10^{-7} \mathrm{~cm}$ it is less than $10^{7}$ years.

For the small-sized members of the ASSB family $\left(r_{\mathrm{d}} / 2<\right.$ $R_{\mathrm{ASSB}}<L_{\mathrm{d}}$, viz. the larger grains and meteoroids) the disintegration due to track-breaking is somewhat slower. In the model of a spherical ASSB, the formation of additional EZs of an elliptic shape, apart from the mentioned EZ shaped as a parabolic segment, is possible on the surface of these bodies. Such zones are formed when the ion track originates in the $\sim r_{\mathrm{d}}$-thick subsurface layer. The average length $a_{\mathrm{e}}$ of such an EZ is $a_{\mathrm{e}} \sim$ $2\left(r_{\mathrm{d}} R_{\mathrm{ASSB}}\right)^{1 / 2}$, and its average width is $b_{\mathrm{e}} \sim 2\left(c r_{\mathrm{d}}\right)^{1 / 2}$ for an invariable depth $c \sim 10^{-7} \mathrm{~cm}$. The erosion rate for these ASSBs can be evaluated from the formula

$$
\begin{aligned}
-\frac{\mathrm{d} M\left(R_{\mathrm{ASSB}}\right)}{\mathrm{d} t} \approx & 8 \pi^{2}\left(R_{\mathrm{ASSB}}-r_{\mathrm{d}}\right)^{2} \cdot J \cdot V \cdot \rho \\
& +32 \pi^{2} r_{\mathrm{d}}^{2} R_{\mathrm{ASSB}}^{3 / 2} \cdot \rho,
\end{aligned}
$$

here $V$ is the EZ volume, the first term corresponds to the parabolic EZ contribution, and the second to the elliptic one. Then in defining the $t_{\mathrm{s}}$ value in this size range we have the following equation

$$
\begin{aligned}
t_{\mathrm{S}}\left(R_{\mathrm{ASSB}}\right) \approx & \frac{3}{8}\left(\pi J \cdot \ln \frac{L_{\mathrm{d}}}{r_{\mathrm{d}}}\right)^{-1} c^{-3 / 2} r_{\mathrm{d}}^{1 / 2} \\
& \times \int_{1}^{R_{\mathrm{ASSB}} / r_{\mathrm{d}}} \frac{x^{2} \mathrm{~d} x}{(x-1)^{2}+b x^{3 / 2}} .
\end{aligned}
$$


For $r_{\mathrm{d}}=10^{-4} \mathrm{~cm}, R_{\mathrm{ASSB}}=10^{-2} \mathrm{~cm}$ and for the multichargedion flux value $J=3 \times 10^{-3} \mathrm{~cm}^{-2} \mathrm{~s}^{-1} \mathrm{sr}^{-1}$, which is characteristic for the near-earth space, the calculations with formula (10) give a value of $t_{\mathrm{s}}$ of about $5 \times 10^{7}$ years. During this period such a particle converts primarily into a micron-sized grain, for which the grinding timescale is minimal (see Fig. 7). On the other hand, the Poynting-Robertson drag is also more effective for micron/submicron grains. Therefore, the process of desintegration of these grains is accompanied by their approaching the Sun.

In the range of sizes $R_{\mathrm{ASSB}}>L_{\mathrm{d}}$ the track-breaking differs little from that in the model of the semi-infinite body with a plane boundary, where the EZ has the typical shape of a parabolic segment with an average mass circa $10^{-15} \mathrm{~g}$. In this case, in finding the surface erosion rate, a relatively simple formula from Kalinichenko et al. (1996) can be used:

$-\frac{\mathrm{d} M\left(R_{\mathrm{ASSB}}\right)}{\mathrm{d} t} \approx 8 \pi^{2} R_{\mathrm{ASSB}}^{2} J \cdot V \cdot \rho$.

Such an ASSB exposed to this rate of track-breaking disintegration reaches a size typical for ADG during

$t_{\mathrm{s}}\left(R_{\mathrm{ASSB}}\right) \approx R(2 \pi J V)^{-1}$.

For instance, for $R_{\mathrm{ASSB}}=1 \mathrm{~cm}$, the $t_{\mathrm{S}}$ value estimated with formula (12) is $1.5 \times 10^{9}$ years. However, as some part of even the largest ASSB-surfaces is involved in the formation of elliptical shaped EZ, for a broader size range from $L_{\mathrm{d}}$ to large asteroids, the use of the above formulas (9) and (10) would be more appropriate. Figure 6 shows the calculated dependence of the integral survival time for small-sized ASSBs in the KB versus their initial radii. This dependence accounts for all described varieties of the track-breaking occurring sequentially in different ASSB disintegration phases from the given $R$ to $R_{\min }$.

It should be kept in view, however, that the estimates of $\mathrm{d} M / \mathrm{d} t$ and $t_{\mathrm{s}}$ from this section, despite the DZ concept used, employ a model of surface track-breaking for a quasihomogeneous ASSB material with the density and dynamic tensile strength typical of silicates. For large dust particles and for the larger bodies, which are fluffy aggregates with low density and strength, the model of volumetric track-breaking in a heterogeneous material will be preferable. Therefore, if the acoustic stress wave attenuation is small enough on the way from the energetic track to the surface, the calculated disintegration rate for such bodies can be significantly higher and, accordingly, their survival times much shorter. In turn, this means that large dust particles may convert rapidly into micron-sized and smaller grains, as these are most strongly subject to drag. In such a scenario, the present KB depletion of micron-sized grains looks quite natural, as for the KB evolution period all the relict grains of these sizes may be disintegrated due to the studied mechanism. The observable micron-sized grains were apparently formed by relatively rare recent collisions, and continuous track-breaking conversion of larger bodies, these grains thus not being subject to the drag to the full extent.

When analyzing the possibilities for ADG observation in the solar system, one should take into consideration the ADG peculiarities stemming from the simulated track-breaking

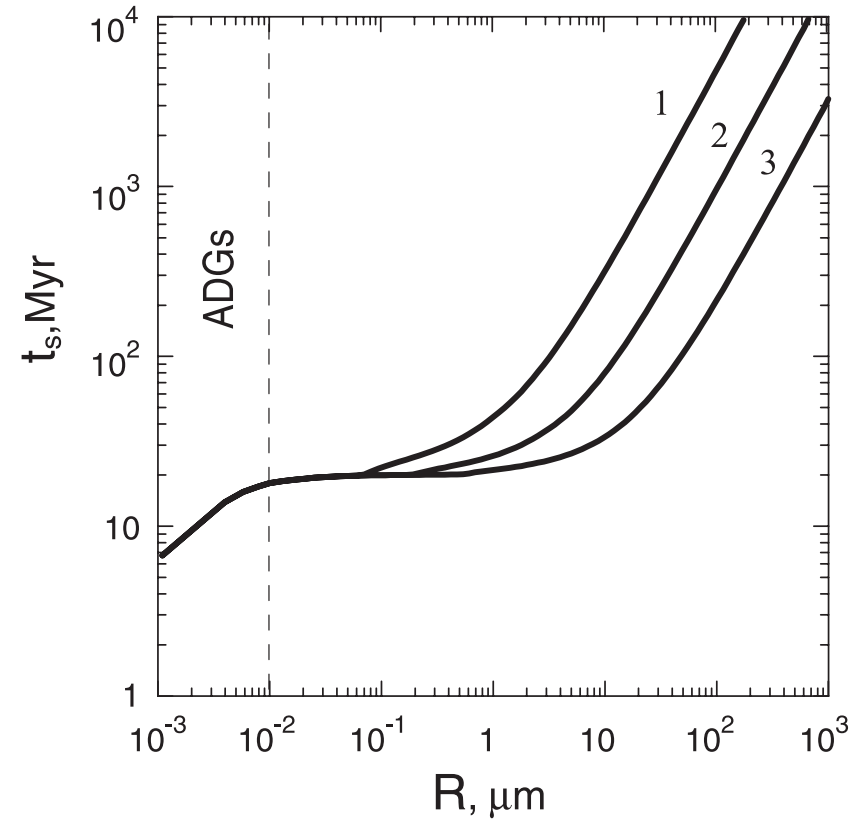

Fig. 6. The theoretical dependence of survival time of the smallest $\mathrm{KB}$ bodies subject to surface ion-enhanced microerosion versus size. The calculations involve particles with the density and dynamic tensile strength typical of monolithic silicates. Curves 1, 2 and 3 are plotted for destruction zone radii of 0.1 microns, 0.3 microns, and 1 micron, respectively.

study. In particular, we may expect the ADGs revealing themselves in far UV and X-ray scattering. The individual ADGs will likely be optically invisible in scattered light at any orientation. On the other hand, if the ADGs become aggregated in clusters, they can be disguised as dust particles of larger masses in the observations in scattered light.

At the same time, possible spatial inhomogeneity of the ADG population in the heliosphere may be revealed in their thermal emission spectrum. The spectrum and intensity of the ADG thermal emission in the solar system are not quite clear yet. Recently, however, the infrared emission from silicon and graphite interstellar nanoparticles of similar mass has been studied theoretically in Li \& Draine (2001, 2002).

The escape processes in this region of the solar system caused by collisions with interstellar particles, and PoyntingRobertson and plasma drags, contribute much to the KB mass loss. As the micron/submicron-sized and smaller grains suffer most from the processes mentioned (Jewitt \& Luu 1997), the KB apparently loses these particles before they are completely track-broken. Recall here that the persistent ion-enhanced erosion may whittle down relatively large dust particles rather rapidly (with characteristic time of about $\sim 10^{6}$ years), thus transferring them to the size range typical of the grains that escape most easily. Figure 7 shows the timescales for trackbreaking in comparison with the known escape processes. It is clearly seen that the mechanism under discussion can be the major reason for small-grain depletion of the KB. With respect to Fig. 7, it should be noted that the graph corresponding to the Poynting-Robertson drag was plotted using the geometrical optics approximation and without taking into account 


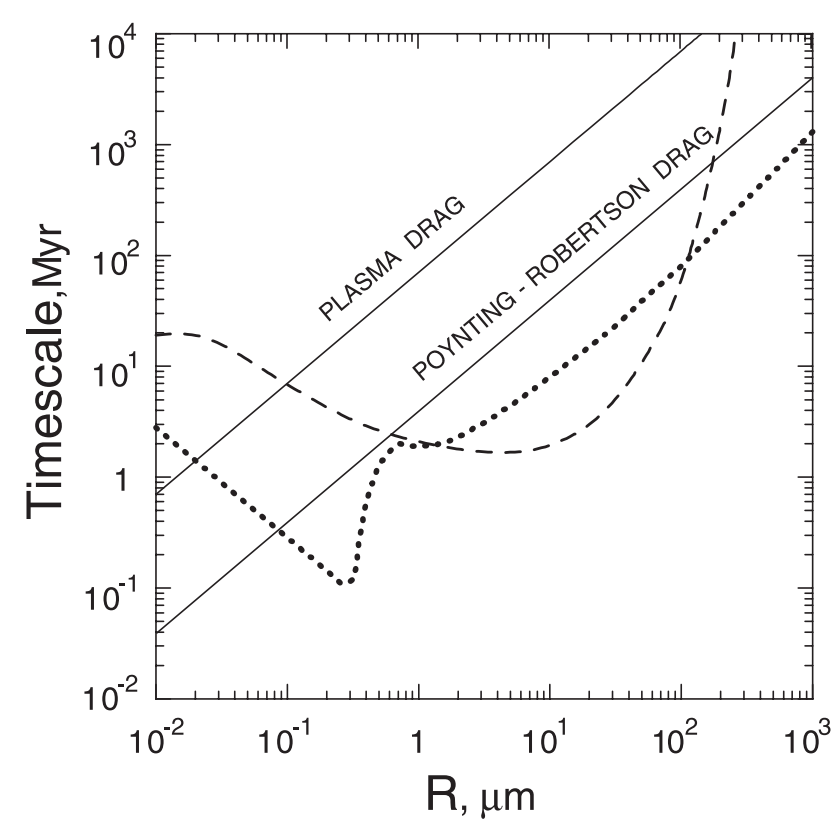

Fig. 7. Comparison of the track-breaking grinding timescale (the period in which the ASSB radius decreases by a factor of $e$, calculated for $r_{\mathrm{d}}=0.3$ microns, dotted line) with that of different escape mechanisms taken from Jewitt \& Luu (1997) for KB bodies in the size-range 0.01 to $10^{3}$ microns. The dashed line indicates the timescale corresponding to the collisions with the interstellar grains. The minimum on our curve lies within the "blow out" region indicated in Fig. 11 of Jewitt (1999).

the sharp decrease in light pressure for the grains smaller that 0.1 micron. It can also bee seen from Fig. 7 that the collisional timescale exceeds that of track-breaking for a wide range of grain sizes, even assuming that the ion-stimulated microerosion mechanism only affects the surface and yields relatively little ADG. For the beta-meteoroids, as this figure shows, the track-breaking timescale becomes minimal and quite competitive with the known timescales even in the event when these dust grains consist of a notably strong monolithic material.

If the track-broken ADGs are electrically charged and if the charge is not neutralized for another $10^{3}-10^{4}$ years, then the attogram dust population can be blown from the KB to the periphery of the heliosphere even more rapidly. The uncharged ADGs and especially those aligned by the solar light pressure will most likely be subject to collisional drag and evaporation only and may remain in the KB for about $10^{7}$ years.

Those grains that have sizes more than $r_{\mathrm{d}} / 2$ and stay in the $\mathrm{KB}$ long enough can produce a large amount of ADG and, thus, be the main cause of the KB mass depletion when the trackbreaking and drag mechanisms are acting together (see scheme in Fig. 8). Therefore, expression (9) can be used for estimating the total disintegration rate $\mathrm{d} M_{\mathrm{KB}} / \mathrm{d} t$ for the $\mathrm{KB}$ mass loss

$$
-\frac{\mathrm{d} M_{\mathrm{KB}}}{\mathrm{d} t} \approx 8 \pi^{2} \int_{R_{1}}^{R_{2}}\left(J\left(R-r_{\mathrm{d}}\right)^{2} V \rho-4 J(c R)^{3 / 2} \rho\right) A_{\mathrm{KB}} R^{-4} \mathrm{~d} R,
$$

where $A_{\mathrm{KB}}=3.75 \times 10^{25} \mathrm{~cm}^{3}$ is the size distribution constant determined from the known estimates of the number of large $\mathrm{ASBB}$ in the $\mathrm{KB}, R_{1}=10 \mu \mathrm{m}, R_{2}=300 \mathrm{~km}$. By integration and substitution of the above used parameter values we obtain $\mathrm{d} M_{\mathrm{KB}} / \mathrm{d} t \sim 5 \times 10^{16} \mathrm{~kg} \mathrm{year}^{-1}$. Hence it follows that, in the $\mathrm{KB}$ evolution of about $5 \times 10^{9}$ years the KB mass loss, owing only to track-breaking of large particles and larger bodies and to the subsequent ADG escape and evaporation, may exceed by two or more orders the presently estimated KB mass that is in the solid phase. In addition to the above-discussed scenario of the conversion of large particles into grains that can be blown out more easily, this also shows that our approach is able to cast light on the dramatic history of the KB mentioned in Jewitt (1999), which led to only one percent of the initial mass of the KB being left by now.

In the light of observational data on circumstellar dust disks in the Galaxy it looks logical that dust should disintegrate by track-breaking and that a significant part of the dust component of the KB should become invisible. Actually, only a few disks are directly observable, in particular, for Vega-like stars. There is a much greater number of stars suspected of having circumstellar disks based on infrared excess observations. However, this constitutes only a small fraction off all possible candidates, which can be roughly estimated as being $10^{8}$ stars. Additionally, all known stars with circumstellar disks are younger than the Sun. From this we can assume that, at least for the solar-age or older stars, a significant part of the circumstellar dust component already passed the stage of trackbreaking disintegration.

It should be noted that, since the intensity of blowing out due to the light pressure increases rapidly with the increase in star luminosity, the circumstellar and protoplanetary dust disks of young stars can be considered as the high-power sources of ultrafine interstellar dust rather than the solar system. The rate of galactic ADG repopulation in this manner may essentially exceed $10^{20}$ tons per year.

If we consider that ordinary-size interstellar dust is also subject to track-breaking disintegration, the more so as in the interstellar medium there is less weakening of the cosmic ray energy spectrum cutoff, the rate mentioned above must be a lower estimate. Moreover, the studied process of conversion of larger space bodies into the smaller ones, as well as into ADGs, is characteristic, apparently, of all the regions of the Galaxy, and of the other galaxies, where the primary cosmic rays can penetrate and where solid matter exists.

\section{Summary}

Brittle microerosion of ASSB surfaces due to the trackbreaking mechanism, where the ASSBs are bombarded by multicharged cosmic-ray ions, may be an important source of the ADG population in the solar system. A quantitative analysis of this mechanism made for simple models, which permit numerical evaluations of the expected parameters of the ADGs produced by various ASSB types, makes it possible to explain a number of interesting observational data. Among these is a significant surplus of ADGs in the cometary environment. Another example may be the present state of the Kuiper Belt with its depletion of micron-sized dust particles and the loss of the bulk of its initial mass (in the solid phase) in evolution. Probably, such a mass loss is typical for the circumstellar disks 


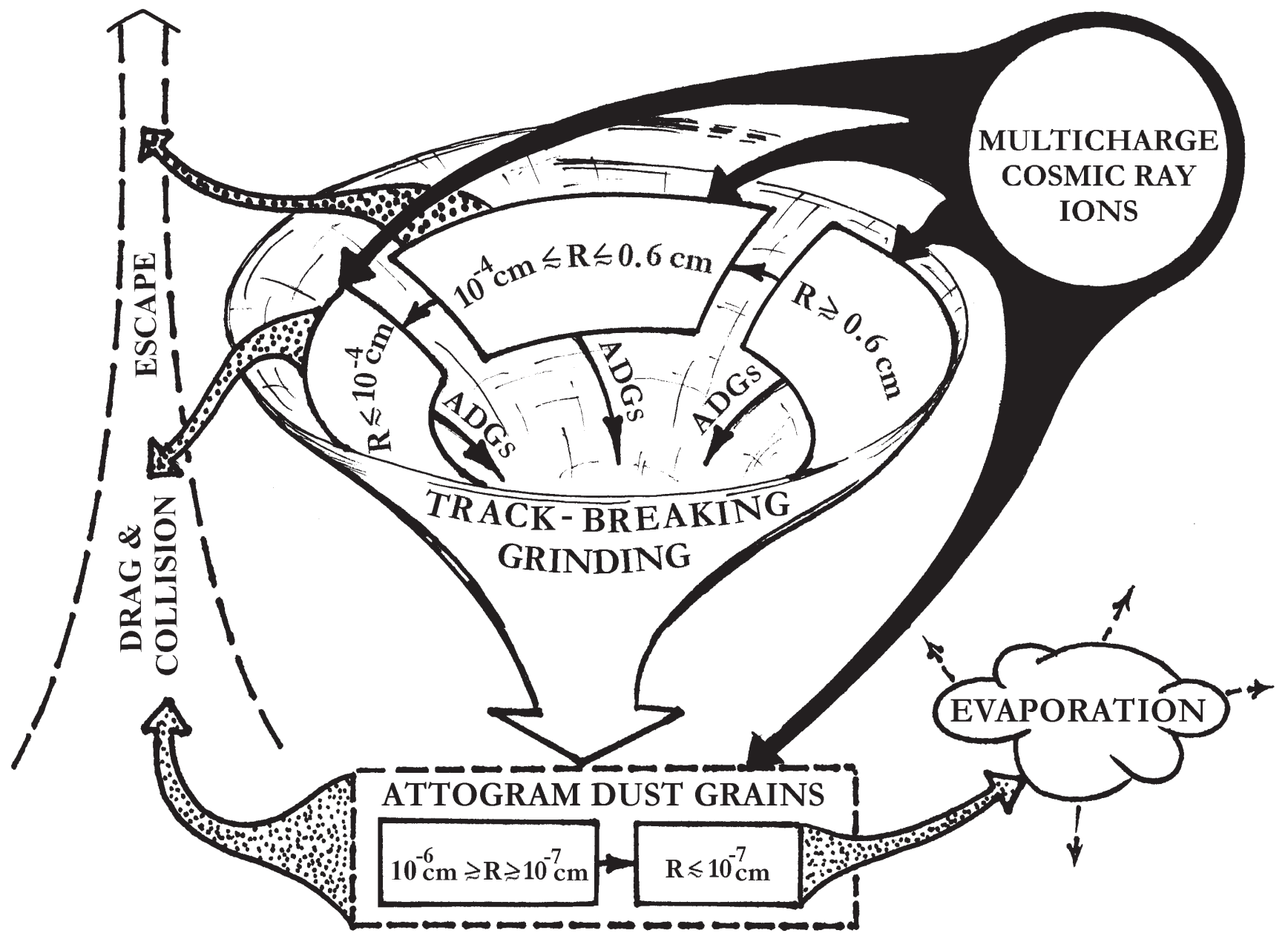

Fig. 8. Schematic explanation of the KB mass loss. The track-breaking grinding and conversion of relatively large dust grains into a micron/submicron-sized population with continuous ADG formation followed by drag and collisional escape are the most productive channels for these losses in the studied model. Such processes might result in the KB losing the bulk of its mass during its evolution.

and planetary/protoplanetary systems of single main-sequence stars.

The ADG production resulting from track-breaking purely due to microerosion of bodies with a cross-section of $20 \mathrm{mi}-$ crons and larger, and only in the Kuiper Belt, is estimated to exceed $5 \times 10^{11}$ tons per year. For the whole solar system, ionenhanced ASSB microerosion owing to track-breaking can be assumed to be the mechanism that is chiefly responsible for the formation of ADG mainly from the smallest bodies, i.e. from the micron/submicron-sized dust particles. The comets, whose nuclei may accumulate attogram grains in their formation, may contribute substantially to the attogram dust population too, especially during the phases of intensive evaporation of volatiles. The ADGs deposited on large asteroids also may escape from the parent body under impacts and thus repopulate.

The proposed ADG forming mechanism also makes it possible to make some obsevational predictions, in particular, concerning the expected range of properties for the ice-rich ASSB of rock type and remote from the Sun that should show a quasisymmetric dust cocoon with an excess of scattered radiation in the short-wavelength region of the spectrum. The ADGs can be generated by a rather large quantity of potential sources. On the other hand, they are highly dynamic (especially if they are charged) and have a comparatively long survival time. This lets us assume that the attogram dust population can show up not only in the vicinity of near Sun/Earth comets and asteroids but in the periphery of the solar system and heliosphere as well. For instance, the ADGs can show up as an optically invisible non-homogeneity of the background radiation.

Generally, a large number of aspects of the problem concerning the ADG population in space, both discussed in this paper and related ones, requires a more detailed and separate study. In this regard, the expected results of the STARDUST mission can be very important.

Acknowledgements. The authors thank Prof. M. Greenberg who is no longer with us but who was the first well-known expert to support this study. It is our pleasure to thank B. Gustafson, M. A'Hearn, T. Kostiuk, M. Mumma, J. Kerrige, T. Owen, M. Hanner, and V. Krasnopolsky for encouraging our work and stimulating discussions. We also wish to express our appreciation to D. Hamilton, V. Zubco, V. Tishkovets, and to all participants of local and overseas seminars for useful remarks. We also thank the anonymous referees for very helpful and constructive remarks. 


\section{References}

Eichhorn, G. 1975, P\&SS, 23, 1519

Gurnett, D. A., Ansher, J. A., Kurth, W. S., \& Granroth, L. U. 1997, Geo. Res. Lett., 24, 3125

Greenberg, J. M. 1998, A\&A, 330, 375

Hutcheon, I. D. 1975, J. Geophys. Res., 80, 4471

Jewitt, D. 1999, Annu. Rev. Earth Planetary Sci., 27, 287

Jewitt, D., \& Luu, J. X. 1997, in ASP Conf. Ser., 122, ed. Y. J. Pendleton \& A. Tielens (San Francisco), 335

Kalinichenko, A. I., Vasilyev, V. P., \& Vasilyev, S. V. 1996, Earth, Moon, and Planets, 74, 17

Krasnopolsky, V. 1997, Icarus, 128, 368

Lamy, P. L., Grün, E., \& Perrin, J. M. 1987, A\&A, 187, 767

Li, A., \& Draine, B. T. 2001, AJ, 554, 778

Li, A., \& Draine, B. T. 2002, AJ, 564, 803

Lisse, C. M., Dennerl, K., Englhauser, J., et al. 1996, Science, 274, 205

Lisse, C. M., Dennerl, K., Englhauser, J., et al. 1997, Earth, Moon, and Planets, 77, 283
Luu, J. X. 1994, PASP, 106, 425

Meech, K. J., Buie, M. W., Samarasinha, N., et al. 1994, DPS Abstr., BAAS, 26, 1152

Sagdeev, R. Z., Evlanov, E. N., Fomenkova, M. N., et al. 1989, Adv. Space. Res., 9, 263

Tielens, A. G. G. M. 1999, in Formation and Evolution of Solid in Space, ed. J. M. Greenberg \& A. Li (Dordrecht, Boston, London: Kluver Acad. Publ.), Proc. NATO Adv. Study Inst. on Formation and Evolution of Solid in Space, Erice, Italy, 10-27 March, 1997, 331

van Nes, P. 1986, Ruimtevaart, 35, 1 (in Dutch)

Vasilyev, V. P., Kalinichenko, A. I., \& Vasilyev, S. V. 1998, in Proc. Conf. on the High Energy Radiation Background in Space, Snowmass, Colorado, July 21-25, 1997, IEEE/Nuclear and Plasma Sciences Society, 86

Utterback, N. G., \& Kissel, J. 1990, AJ, 100, 1315

West, R. M. 1991, A\&A, 241, 635 